\title{
Unified model for network dynamics exhibiting nonextensive statistics
}

\author{
Stefan Thurner 1, * Fragiskos Kyriakopoulos ${ }^{1,2}$, and Constantino Tsallis ${ }^{3,4}$ : \\ ${ }^{1}$ Complex Systems Research Group, HNO Medical University of Vienna, Währinger Gürtel 18-20, A-1090, Austria \\ ${ }^{2}$ Institute of Theoretical Physics, Johannes Kepler University, Altenbergerstrasse 69, Linz, A-4040, Austria \\ ${ }^{3}$ Santa Fe Institute, 1399 Hyde Park Road, Santa Fe, NM 87501, USA \\ ${ }^{4}$ Centro Brasileiro de Pesquisas Fisicas, Rua Xavier Sigaud 150, 22290-180 Rio de Janeiro-RJ; Brazil
}

(Dated: July 6, 2018)

\begin{abstract}
We introduce a dynamical network model which unifies a number of network families which are individually known to exhibit $q$-exponential degree distributions. The present model dynamics incorporates static (non-growing) self-organizing networks, preferentially growing networks, and (preferentially) rewiring networks. Further, it exhibits a natural random graph limit. The proposed model generalizes network dynamics to rewiring and growth modes which depend on internal topology as well as on a metric imposed by the space they are embedded in. In all of the networks emerging from the presented model we find $q$-exponential degree distributions over a large parameter space. We comment on the parameter dependence of the corresponding entropic index $q$ for the degree distributions, and on the behavior of the clustering coefficients and neighboring connectivity distributions.
\end{abstract}

PACS numbers: $\quad$ 05.70.Ln, 89.75.Hc, 89.75.-k

\section{INTRODUCTION}

Over the past two decades, nonextensive statistical mechanics has successfully addressed a wide spectrum of nonequilibrium phenomena in non-ergodic and other complex systems [1, 2]. Recently, it has also entered the field of networks $[3,4,4,6,6,7,8,9,10]$. Nonextensive statistical mechanics is a generalization of Boltzmann-Gibbs (BG) statistical mechanics. It is based on the entropy

$$
S_{q} \equiv \frac{1-\int d x[p(x)]^{q}}{q-1} \quad\left(S_{1}=S_{B G} \equiv-\int d x p(x) \ln p(x)\right) .
$$

The extremization of the entropy $S_{q}$ under appropriate constraints [11] yields the stationary-state distribution. This is of the $q$-exponential form, where the $q$-exponential function is defined as

$$
e_{q}^{x} \equiv[1+(1-q) x]^{1 /(1-q)},
$$

for $1+(1-q) x \geq 0$, and zero otherwise (with $\left.e_{1}^{x}=e^{x}\right)$. The tail exponent $\gamma \equiv 1 /(q-1)$ characterizes the asymptotic power-law distribution.

Since the very beginning of the tremendous recent modeling efforts of complex networks it has been noticed that degree distributions asymptotically follow power-laws [12], or even exactly $q$-exponentials [13]. The model in 12. describes growing networks with a so-called preferential attachment rule, meaning that any new node $i$ being added to the system links itself to an already existing node $j$ in the network with a probability that is proportional to the degree $k_{j}$ of node $j$. In [13] this model was extended to also allow for preferential rewiring. The analytical solution to the model has a $q$-exponential as a result, with the nonextensivity parameter $q$ being fixed uniquely by the model parameters. Recently in [4] preferential attachment networks have been embedded in Euclidean space, where the attachment probability for a newly added node is not only proportional to the degrees of existing nodes, but also depends on the Euclidean distance between nodes. The model is realized by setting the linking probability of a new node to an existing node $i$ to be $p_{\text {link }} \propto k_{i} / r_{i}^{\alpha}(\alpha \geq 0)$, where $r_{i}$ is the distance between the new node and node $i ; \alpha=0$ corresponds to the model in [12] which has no metrics. The analysis of the degree distributions of the resulting networks has exhibited [4] $q$-exponentials with a clear $\alpha$-dependence of the nonextensivity parameter $q$. In the large $\alpha$ limit, $q$ approaches unity, i.e., random networks are recovered in the Boltzmann-Gibbs limit. In an effort to understand the evolution of socio-economic networks, a model was proposed in [7] that builds upon [13] but

*Electronic address: thurner@univie.ac.at

${ }^{\dagger}$ Electronic address: tsallis@santafe.edu 
introduces a rewiring scheme which depends on the internal network distance between two nodes, i.e., the number of steps needed to connect the two nodes. The emerging degree distributions have been subjected to a statistical analysis where the (null) hypothesis of $q$-exponentials could not be rejected.

It has been found that networks exhibiting degree distributions compatible with $q$-exponentials are not at all limited to growing and preferentially organizing networks. A model for nongrowing networks which was recently put forward in [5] also unambiguously exhibits $q$-exponential degree distributions. This model was motivated by interpreting networks as a certain type of 'gas' where upon an (inelastic) collision of two nodes, links get transfered in analogy to the energy-momentum transfer in real gases. In this model a fixed number of nodes in an (undirected) network can 'merge', i.e., two nodes fuse into one single node, which keeps the union of links of the two original nodes; the link connecting the two nodes before the merger is removed. At the same time a new node is introduced to the system and is linked randomly to any of the existing nodes in the network [14]. Due to the nature of this model the number of links is not strictly conserved - which can be thought of as jumps between discrete states in some 'phase space'. The model has been further generalized to exhibit a distance dependence as in [4], however $r_{i}$ not being Euclidean but internal distance. Again, the resulting degree distributions have $q$-exponential form.

A quite different approach was taken in [15] where an ensemble interpretation of random networks has been adopted, motivated by superstatistics [16]. Here it was assumed that the average connectivity $\bar{k}$ in random networks is fluctuating according to a distribution $\Pi(\bar{k})$, which is sometimes associated with a 'hidden-variable' distribution. In this sense a network with any degree distribution can be seen as a 'superposition' of random networks with the degree distribution given by $p(k)=\int_{0}^{\infty} d \lambda \Pi(\bar{k}) \frac{\bar{k}^{k} e^{-\bar{k}}}{k !}$. It was shown in [15], as an exact example, that an asymptotically power-law functional form of $\Pi(\bar{k}) \propto \bar{k}^{-\gamma}$ leads to degree distributions of Zipf-Mandelbrot form, $p(k) \propto \frac{1}{\left(k_{0}+k\right)^{\gamma}}$, which is equivalent to a $q$-exponential $e_{q}^{-k / \kappa}$ with $\kappa \equiv(1-q) k_{0}$ and $q \equiv 1+1 / \gamma$. Very recently a possible connection between small-world networks and the maximum $S_{q}$-entropy principle, as well as to the hidden variable method [15], has been noticed in [9].

In yet another view, networks have recently been treated as statistical systems on a Hamiltonian basis [17, 18, 19, 20]. It has been shown that these systems show a phase transition like behavior [18], along which networks structure changes. In the low temperature phase one finds networks of 'star' type, meaning that a few nodes are extremely well connected resulting even in a discontinuous $p(k)$; in the high temperature phase one finds random networks. Surprisingly, for a special type of Hamiltonians networks with $q$-exponential degree distributions emerge right in the vicinity of the transition point [20].

Given the above characteristics of networks and the fact that a vast number of real-world and model networks show asymptotic power-law degree distributions, it seems almost obvious to look for a deeper connection between networks and nonextensive statistical physics. The purpose of this work is to show that various model types can be unified into a single dynamic network-formation model, characterized by a reasonably small number of parameters. Within this parameter space, all networks seem to be compatible with $q$-exponential degree distributions.

\section{MODEL}

The following model is a unification and generalization of the models presented in [4, 5]. The model in [4] captures preferential growing aspects of networks embedded into a metric space, while [5] introduces a static, selforganizing model with a sensitivity to an internal metric (chemical distance, Diekstra distance). The rewiring scheme there can be thought of having preferential attachment aspects in one of its limits [14] (see below), but has none in the other limit.

\section{A. Network model}

The network evolves in time as described in [4]: At $t=1$, the first node $(i=1)$ is placed at some arbitrary position in a metric space. The next node is placed isotropically on a sphere (in that space) of radius $r$, which is drawn from a distribution $P_{G}(r) \propto 1 / r^{\alpha_{G}}\left(\alpha_{G}>0, G\right.$ stands for growth. To avoid problems with the singularity, we impose a cutoff at $r_{\min }=1$. The second node is linked to the first. The third node is placed again isotropically on a sphere with random radius $r \in P_{G}$, however the center of the sphere is now the barycenter of all the pre-existing nodes. From the third added node on, there is an ambiguity where the newly positioned node should link to. We choose a generalized preferential attachment process, meaning that the probability that the newly created node $i$ attaches to a previously existing node $j$ is proportional to the degree $k_{j}$ of the existing node $j$, and on the metric (Euclidean) 

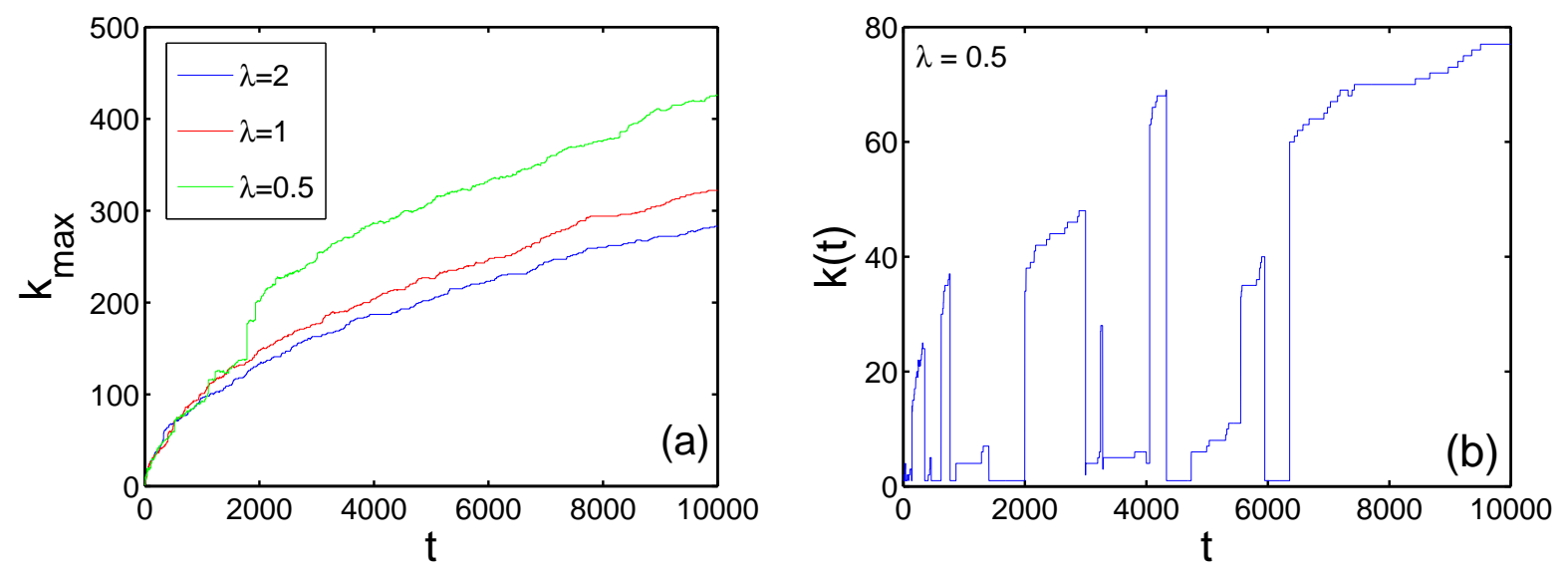

FIG. 1: Time evolution of the degree of the best connected node (a) and of a randomly chosen node (b) for the parameters, $N=10000, \alpha_{A}=0, \alpha_{M}=0$.

distance between $i$ and $j$, denoted by $r_{i j}$. In particular the linking probability is

$$
p_{i j}^{A}=\frac{k_{j} / r_{i j}^{\alpha_{A}}}{\sum_{j=1}^{N(t)-1} k_{j} / r_{i j}^{\alpha_{A}}},
$$

where $N(t)$ is the number of nodes at time $t$. It is not necessary that at each time step only one node is entering the system, so we immediately generalize that a number of $\bar{n}$ nodes are produced and linked to the existing network with $\bar{l}$ links per time step. Note that $\bar{n}$ and $\bar{l}$ can also be random numbers from an arbitrary distribution. For simplicity and clarity we fix $\bar{n}=1$ and $\bar{l}=1$.

After every $\lambda$ timesteps, a different action takes place on the network. At this timestep the network does not grow but a pair of nodes, say $i$ and $j$, merge to form one single node [14]. This node keeps the name of one of the original nodes, say for example $i$. This node now gains all the links of the other node $j$, resulting in a change of degree for node $i$ according to

$$
\begin{aligned}
& k_{i} \rightarrow k_{i}+k_{j}-N_{\text {common }} \quad, \quad \text { if }(i, j) \text { are not first neighbors } \\
& k_{i} \rightarrow k_{i}+k_{j}-N_{\text {common }}-2 \quad, \quad \text { if }(i, j) \text { are first neighbors }
\end{aligned}
$$

where $N_{\text {common }}$ is the number of nodes, which shared links to both of $i$ and $j$ before the merger. In the case that $i$ and $j$ were first neighbors before the merger, i.e., they had been previously linked, the removal of this link will be taken care of by the term -2 in Eq. (4). The probability that two nodes $i$ and $j$ merge can be made distance dependent, as before. In particular to stay close to the model in [5], we chose the following procedure. We randomly choose node $i$ with probability $\propto 1 / N(t)$ and then choose the merging partner $j$ with probability

$$
p_{i j}^{M}=\frac{d_{i j}^{-\alpha_{M}}}{\sum_{j} d_{i j}^{-\alpha_{M}}} \quad\left(\alpha_{M} \geq 0\right)
$$

where $d_{i j}$ is the shortest distance (path) on the network connecting nodes $i$ and $j$; Obviously, tuning $\alpha_{M}$ from 0 toward large values, switches the model from the case where $j$ is picked fully at random $(\propto 1 / N(t))$, to a case where only nearest neighbors of $i$ will have a nonnegligible chance to get chosen for the merger. Note that the number of nodes is reduced by one at that point. To keep the number of nodes constant at this timestep, a new node is introduced and linked with $\bar{l}$ of the existing nodes with probability given in Eq. (3).

This concludes the model. Summing up, the relevant model parameters, we have the merging exponent $\alpha_{M}$, the attachment exponent $\alpha_{A}$, controlling the sensitivity of 'distance' in the network, and the relative rate of merging and growing, $\lambda$. The parameters, $\alpha_{G}, \bar{n}, \bar{l}$, and $r_{\min }$ have been found to play no major role in the model.

We simulate this model and record the degrees $k_{i}$, the clustering coefficients $c_{i}$ (defined below), and the nearest neighbor-connectivity $k_{i}^{n n}$, for all individual nodes $i$. From these values we derive distribution functions (as a function of $k$ ). In Fig. 2 typical degree distributions are shown for three typical values of $\lambda$. Obviously, the distribution is 


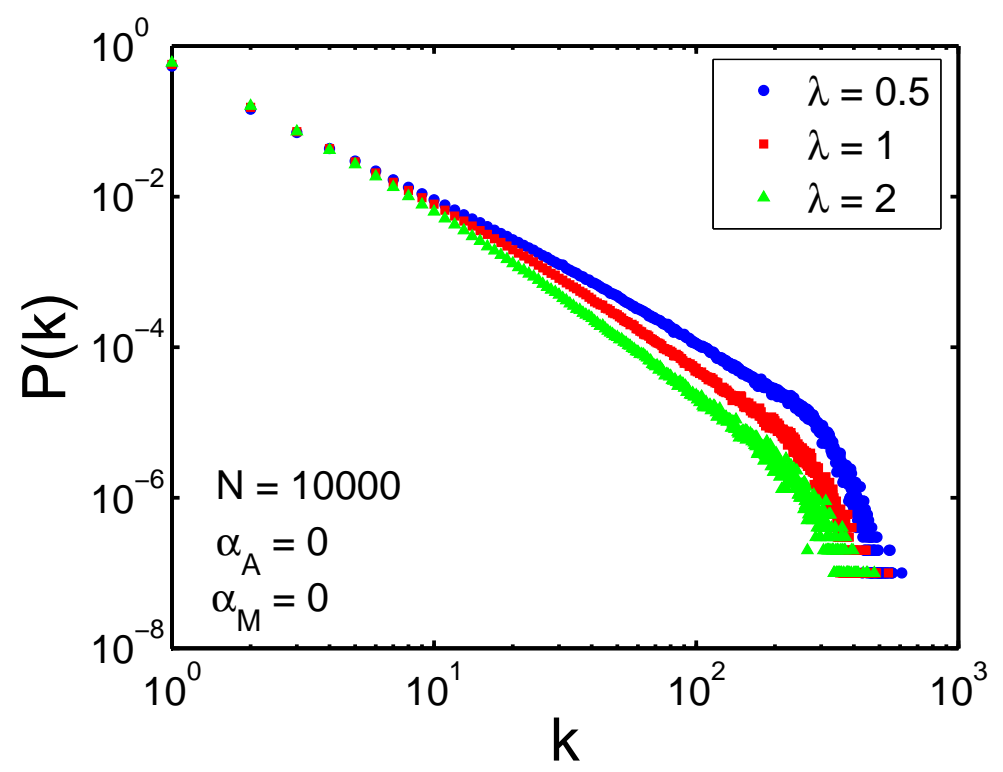

FIG. 2: Degree distribution $P(k)$ (un-normalized) for $N=10000, \alpha_{A}=0, \alpha_{M}=0$ and various values of $\lambda$.

dominated by a power-law decay (see details of the functional form below) ending in an exponential finite size cut-off for large $k$.

The clustering coefficient of node $i, c_{i}$ is defined by

$$
c_{i}=\frac{2 e_{i}}{k_{i}\left(k_{i}-1\right)}
$$

with $e_{i}$ being the number of triangles node $i$ is part of. $c(k)$ is obtained by averaging over all $c_{i}$ with a fixed $k$. It has been noted that $c(k)$ contains information about hierarchies present in networks [21]. For Erdös-Rényi (ER) networks [22], as well as for pure preferential attachment algorithms without the possibility of rewiring, the clustering coefficient $c(k)$ vs. degree is flat. The global clustering coefficient is the average over all nodes $C=\left\langle c_{i}\right\rangle_{i}$. A large global clustering coefficient is often used for identification of small-world structure [23]. The average nearest-neighbor connectivity (of the neighbors) of node $i$ is

$$
k_{i}^{n n}=\frac{1}{k_{i}} \sum_{j \text { neighbor of } i} k_{j}
$$

When plotted as a function of $k, k^{n n}(k)$ is a measure to assess the assortativity of networks. A rising function means assortativity, which is the tendency for well connected nodes to link to other well connected ones, while a declining function signals disassortative structure.

\section{B. Particular instances of the model}

Depending on the variables of the model, known networks result as natural limits.

\section{Soares et al. limit}

For the $\lim \lambda \rightarrow \infty$ we have no merging, and $\alpha_{M}$ is an irrelevant parameter. The model corresponding to this limit has been proposed and studied in [4]. 


\section{Albert-Barabasi limit}

The $\lim \lambda \rightarrow \infty$ and $\lim \alpha_{A} \rightarrow 0$, gets rid of the metric in the Soares et al. model and recovers the original Albert-Barabasi preferential attachment model.

\section{Kim et al. limits}

The limit $\lim \lambda \rightarrow 0$ allows no preferential growing of the network. If at each timestep after every merger a new node is linked randomly with $\bar{l}$ links to the network, the model reported in [5] is recovered. The lim $\lambda \rightarrow 0$ model with $\lim \alpha_{M} \rightarrow 0\left(\lim \alpha_{M} \rightarrow \infty\right)$ recovers the random case (neighbor case) in [14].

\section{NONEXTENSIVE CHARACTERIZATION OF COMPLEX NETWORKS}

There has been a convincing body of evidence that, for a large class of networks, (normalized) degree distributions can be fit by $q$-exponentials,

$$
P(k)=e_{q}^{-(k-1) / \kappa} \quad(k=1,2,3,4, \ldots) \quad,
$$

where the $q$-exponential function is defined in Eq. (2), with $q \geq 1$, and $\kappa>0$ some characteristic number of links. A convenient procedure to perform a two-parameter fit of this kind is to take the q-logarithm of the distribution $P$, defined by $Z_{q}(k) \equiv \ln _{q} P(k) \equiv \frac{[P(k)]^{1-q}-1}{1-q}$. This is done for a series of different values of $q$. The function $Z_{q}(k)$ which can be best fit with a straight line determines the value of $q$, the slope being $-\kappa$.

In Fig. 3] we show the degree distribution for several system sizes together with the $q$-logarithm $Z_{q}(k)$, from which an optimum $q$ and $\kappa$ can be obtained. We conclude that, with good precision, the Ansatz in Eq. (8) for the degree distribution, when seen as a null hypothesis, can not be rejected on the basis of a $\chi^{2}$ statistics for any reasonable significance level, for the system sizes studied.

For actual curve fitting, it is often more convenient to use the cumulative distributions, which can be parametrized by

$$
P(\geq k)=e_{q_{c}}^{-(k-1) / \kappa_{c}} \quad(k=1,2,3,4, \ldots)
$$

On the other hand the corresponding cumulative distribution $P(\geq k)$ is given by (we switch to integral notation for simplicity for a moment)

$$
P(\geq k) \equiv 1-\int_{1}^{k} d k^{\prime} P\left(k^{\prime}\right)=\left[1-\frac{1-q}{\kappa}(k-1)\right]^{\frac{2-q}{1-q}} .
$$

By comparison of coefficients the cumulative parameters are given by

$$
q_{c}=\frac{1}{2-q} \quad \text { and } \quad \kappa_{c}=\frac{\kappa}{2-q}
$$

Whenever we talk about $q$-values corresponding to a cumulative distribution, we use the notation $q_{c}$ and $\kappa_{c}$, where $c$ indicates cumulative.

The remarkable quality of $q$-exponential fits to the degree distributions from the model, reveals a connection [4] of scale-free network dynamics to nonextensive statistical mechanics [1, 2]. To make the point more clear, consider the entropy

$$
S_{q} \equiv \frac{1-\int_{1}^{\infty} d k[p(k)]^{q}}{q-1} \quad\left[S_{1}=S_{B G} \equiv-\int_{1}^{\infty} d k p(k) \ln p(k)\right]
$$

where we assume $k$ as a continuous variable for simplicity. If we extremize $S_{q}$ with the constraints [11]

$$
\int_{1}^{\infty} d k p(k)=1 \quad \text { and } \quad \frac{\int_{1}^{\infty} d k k[p(k)]^{q}}{\int_{1}^{\infty} d k[p(k)]^{q}}=K
$$



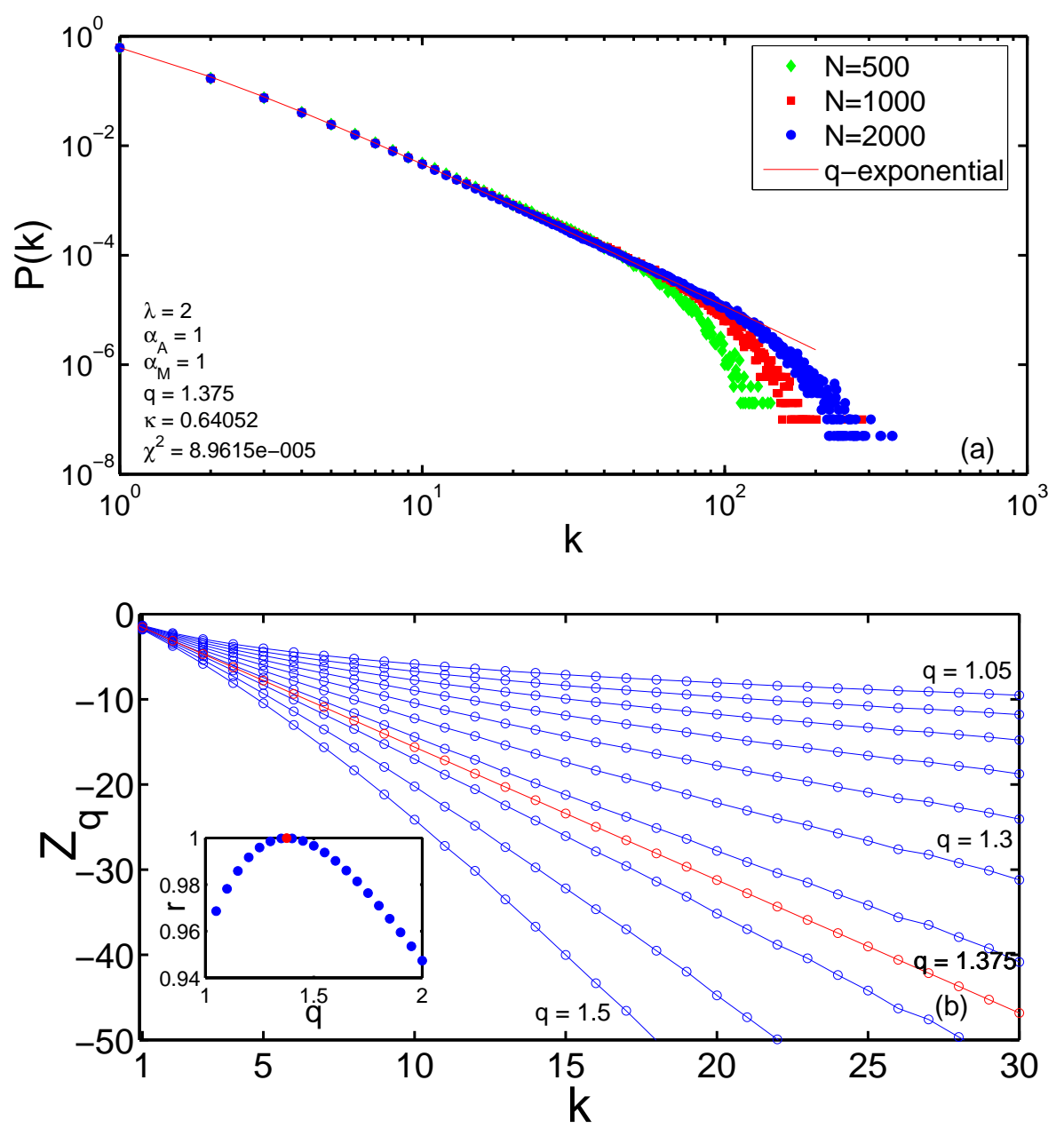

FIG. 3: (a) $P(k)$ for $\lambda=2, \alpha_{A}=1, \alpha_{M}=1$, and various system sizes (symbols). The line is the $q$-exponential fit for $N=2000$. (b) $q$-logarithm of the (normalized) $P(k)$ from (a). The line associated with $q=1.375$ corresponds to an optimal linear fit, i.e. a maximum of the correlation coefficient (inset) of a straight line with $Z_{q}$. The quality of the fit in (a) is given by a standard $\chi^{2}$ statistics.

we obtain

$$
p(k)=\frac{e_{q}^{-\beta(k-1)}}{\int_{1}^{\infty} d k^{\prime} e_{q}^{-\beta\left(k^{\prime}-1\right)}}=\beta(2-q) e_{q}^{-\beta(k-1)} \quad(k \geq 1),
$$

where $\beta$ is determined through Eq. (13). Both positivity of $p(k)$ and the normalization constraint $(13)$ impose $q<2$.

Let us mention that models do exist that can be handled analytically, and which exhibit precisely $q$-exponential degree distributions. Such is the case of [13]. The degree distribution is there presented in the form $p(k) \propto 1 /\left(k+k_{0}\right)^{\gamma}$. This form can be re-written as a $q$-exponential with $q=\frac{\gamma+1}{\gamma}=\frac{2 m(2-r)+1-p-r}{m(3-2 r)+1-p-r}$, where $(m, p, r)$ are parameters of the particular model in [13].

\section{RESULTS}

Realizing the above network model in numerical simulations we compute degree distributions, clustering coefficients, and neighbor connectivity, for a scan over the relevant parameter space, spanned by $\lambda$, $\alpha_{A}$ and $\alpha_{M}$. All following data were obtained from averages over 100 identical network realizations with a final $N\left(t^{\text {max }}\right)=1000$; for finite size checks we have included runs with $N\left(t^{\max }\right)=500$ and 2000 . The reason for these relatively modest network sizes is 
that, at every timestep, all network distances have to evaluated. The remaining parameters have been checked to be of marginal importance and have been fixed to $\alpha_{G}=1, \bar{n}=1$ and $\bar{l}=1$.
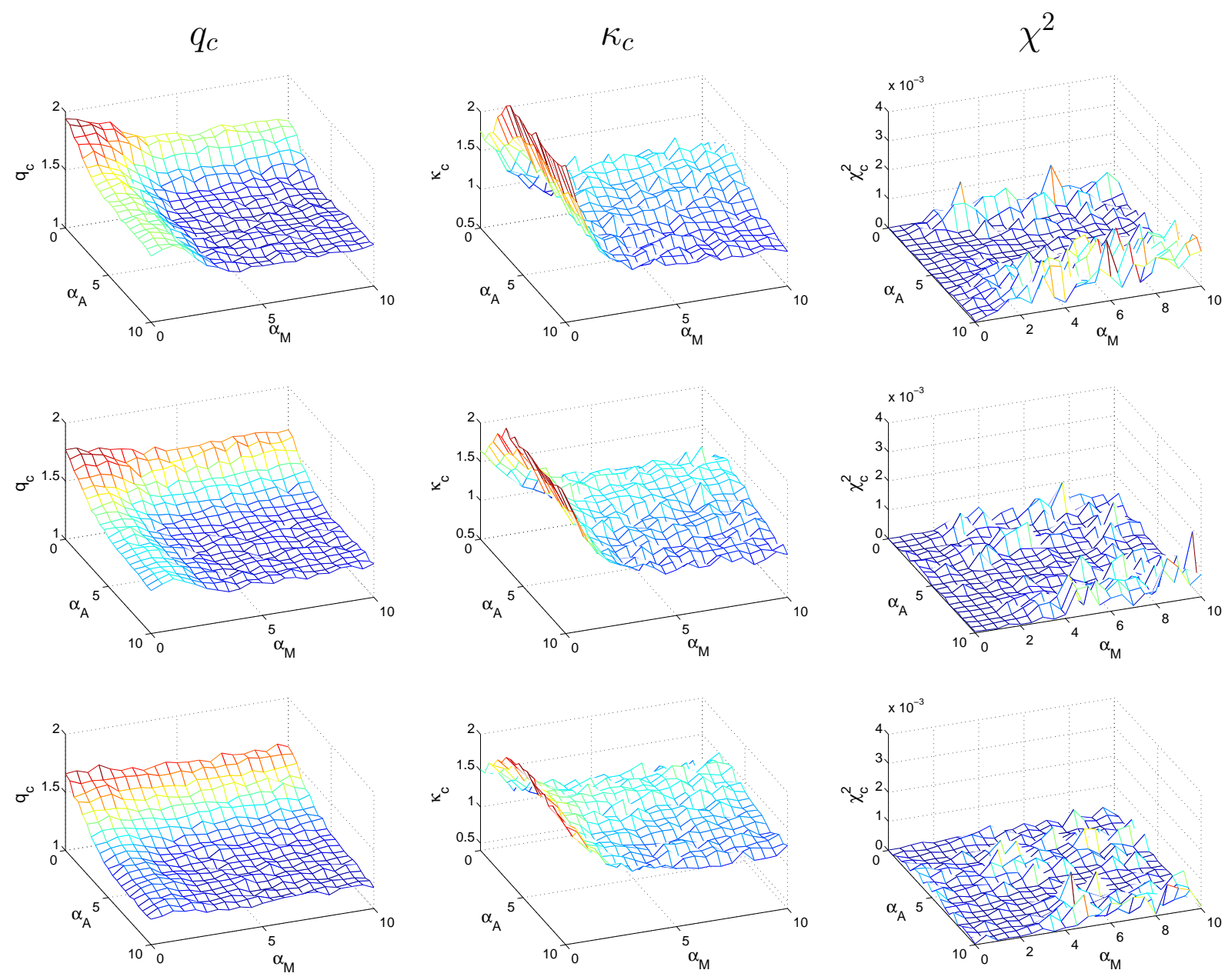

FIG. 4: $q_{c}$ and $\kappa_{c}$ values from $q$-exponential fits to the cumulative degree distributions $P(>k)$ for $\alpha_{G}=1, N=1000$, and $\lambda=0.5$ (top), $\lambda=1$ (middle), $\lambda=2$ (bottom). The fit-quality is given by the $\chi^{2}$ value per degree of freedom.

The fitted values for the nonextensivity index $q_{c}$ and the characteristic degree $\kappa_{c}$ are shown in Fig. 4 over the parameter space. From top to bottom three values of $\lambda$ are shown. The $q_{c}$ index is declining in all three parameters, $\alpha_{A}, \alpha_{M}$, and $\lambda$. It eventually converges to a plateau in the $\alpha_{A}-\alpha_{M}$-plane. The height of the plateau slowly decreases with higher $\lambda$, but remains above $1 ; q_{c}=1$ corresponds to the exponential (ER) case. For low $\alpha_{M}$ there is a maximum of $\kappa_{c}$ at about $\alpha_{A} \sim 3$; For larger $\alpha_{M}$ a plateau is forming for all $\alpha_{A}$. This plateau remains constant as a function of $\lambda$. The quality of the $q$-exponential fit is demonstrated by the $\chi^{2}$ test statistics per degree of freedom.

As in [5] we observe a finite size effect in the data. In Fig. [5 (a) we show the dependence of the degree distribution parameters as a function of $\alpha_{M}$ for different system sizes for a fixed $\alpha_{A}=5$, and $\lambda=2$. The fits for $\kappa_{c}$ are shown in Fig. [5 (b).

We now turn to the clustering and neighbor connectivity of the emerging networks. In Fig. [6 we show the clustering coefficient $c$ and the average neighbor connectivity $k^{n n}$ as a function of $k$. For both quantities, the functional form of the decline with $k$ is well fit with a 2-parameter exponential fit, $\exp \left(-\epsilon_{1} k+\epsilon_{2}\right)$.

In Fig. 7 we show the fit parameters $\epsilon_{1}$ for $c(k)$, (a), and $k^{n n}(k),(\mathrm{b})$, for $\lambda=0.5$. For larger $\lambda$ the clustering coefficients become drastically smaller, as expected for the $\lambda \rightarrow \infty$ and $\alpha_{A} \rightarrow 0$ limit. Fits for $\alpha_{A}>5$ and $\alpha_{M}>5$ become increasingly noisy and are omitted from the figure.

In Fig. 8 we compare the global clustering coefficients from our model, with those obtained from a random graph with the same dimensions (same number of nodes and links). For the Erdös-Renyi random graph the clustering coefficient is $C_{\text {rand }}=\langle k\rangle / N-1$. The comparison makes clear that there is almost no attachment effects for $\alpha_{A}>3$ (i.e., negligible dependence from $\alpha_{A}$ ), and a strong dependence on $\alpha_{M}$ and $\lambda$, as expected. 

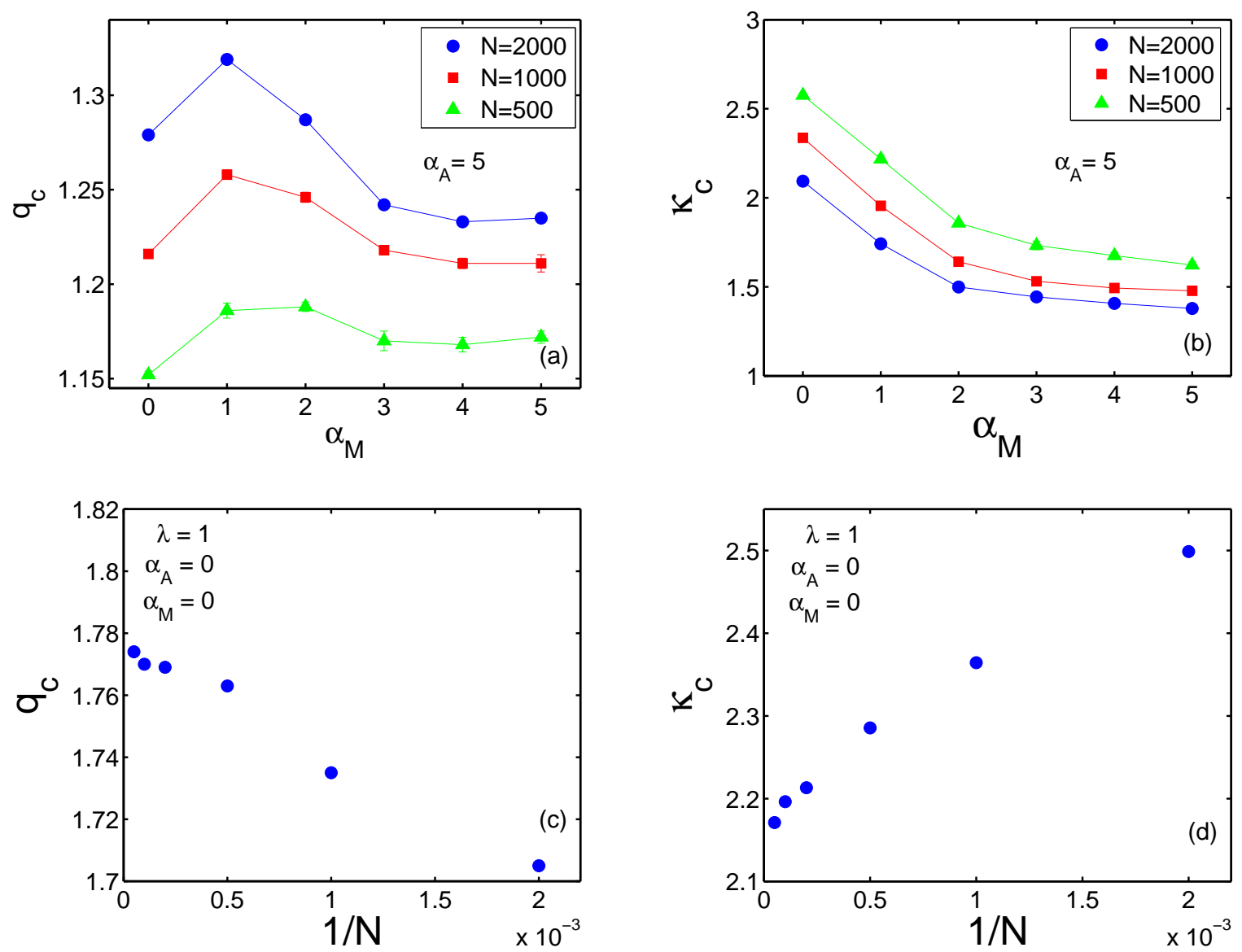

FIG. 5: (a) $q_{c}$ values of 3 system sizes for $\lambda=2, \alpha_{A}=5$, and $\alpha_{M}$ ranging from 0 to 5 . (b) same for $\kappa_{c}$. (c) and (d) show the same parameters as a function of network size $N$, for $\lambda=1, \alpha_{A}=\alpha_{M}=0$. For these parameters networks up to a size of $N=20000$ were possible.

\section{DISCUSSION}

We have introduced a general network formation model which is able to recover, as particular instances, a large class of known network types. We checked that, to a very good approximation, the resulting degree distributions exhibit $q$-exponential forms, with $q>1$. While a full theory of how complex networks are connected to $q \neq 1$ statistical mechanics is still missing, we provide further evidence that such a relation does indeed exist. For example, if we associate a finite fixed energy or "cost" to every bond, and associate with each node half of the energy corresponding to its bonds (the other half corresponding to the other nodes linked by those same bonds), then the degree distribution can be seen as an energy distribution of the type emerging within nonextensive statistical mechanics. It might well be that the full understanding of this relation arises from the discrete nature of networks. The importance of appropriate values of $q \neq 1$ for systems 'living' in topologies with a vanishing Lebesgue measure has been pointed out before [2]. This possibly makes phase space for certain nonextensive systems look like a network itself. In this view the basis of nonextensive systems could be related to a network-like structure of their 'phase space', explaining the ubiquity of $q$-exponential distribution functions in the world of networks.

Let us end by pointing out that, in variance with frequent such statements in the literature, the present model neatly illustrates that never ending growth is not necessary for having networks that are (asymptotically) scale-free. Indeed, $q$-exponential degree distributions do emerge for large enough networks which do not necessarily keep growing. 

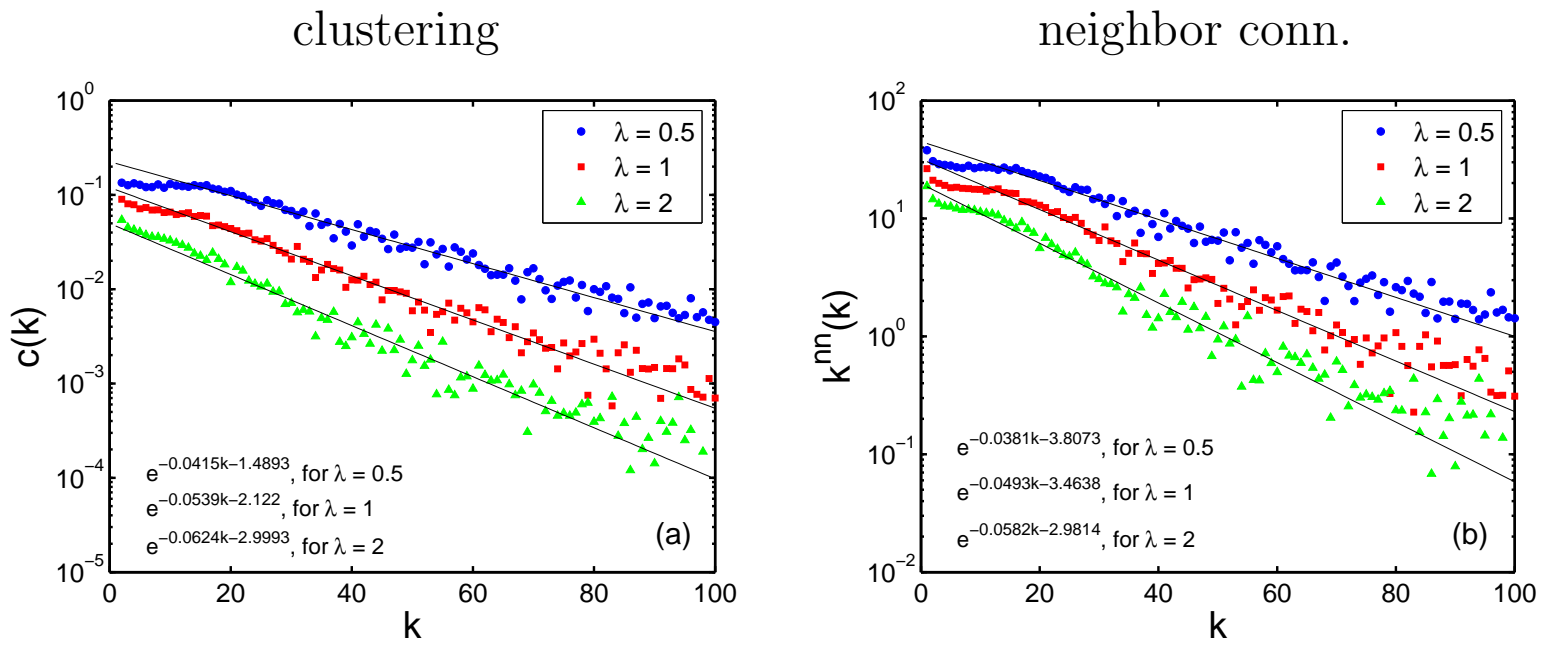

FIG. 6: (a) Clustering coefficient $c(k)$ and (b) average nearest neighbor degree $k^{n n}$, for $\lambda=0.5,1,2$ and a fixed $\alpha_{A}=\alpha_{M}=0$, for $N=1000$. Averaging was done over 100 realizations.

\section{clustering}

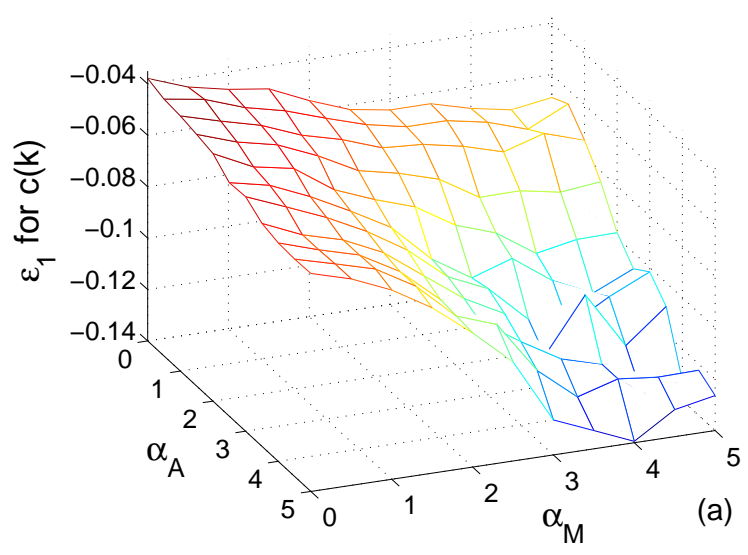

neighbor conn.

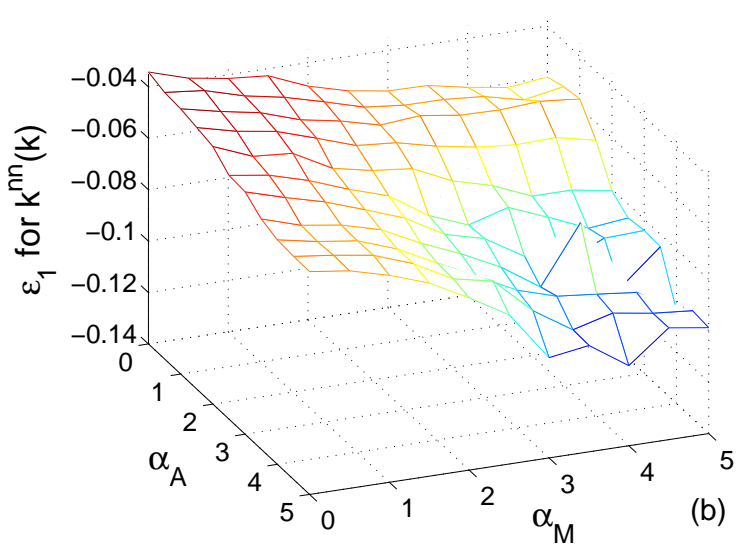

FIG. 7: Exponential decay constants $\epsilon_{1}$ for $c(k)$ (a) and $k^{n n}(k)$ (b) over $\alpha_{A}$ and $\alpha_{M}$ for $\lambda=0.5$. The fit range was $k \in[1,100]$ and averages over 100 independent configurations have been taken. Fits for $\alpha_{A}>5$ and $\alpha_{M}>5$ become statistically insignificant.

\section{Acknowledgments}

S.T. acknowledges support from the Austrian Science Foundation FWF project P19132 and would like to thank the SFI and in particular J.D. Farmer for their great hospitality and support in 2005, where this work was initiated. C.T. acknowledges partial support from Pronex, CNPq and Faperj (Brazilian agencies).

[1] C. Tsallis, J. Stat. Phys 52, 479 (1988).

[2] M. Gell-Mann and C. Tsallis, eds. Nonextensive Entropy - Interdisciplinary Applications (Oxford University Press, New York, 2004); C. Tsallis, M. Gell-Mann and Y. Sato, Proc. Natl. Acad. USA 120, 15377 (2005).

[3] G. Wilk and Z. Wlodarczyk, Acta Phys. Pol. B 35, 871 (2004).

[4] D.J.B. Soares, C. Tsallis, A.M. Mariz, and L.R. da Silva, Europhys. Lett. 70, 70 (2005).

[5] S. Thurner and C. Tsallis, Europhys. Lett. 72, 197-204 (2005).

[6] S. Thurner, Europhysics News 36, 218-220 (2005). 


\section{Model}
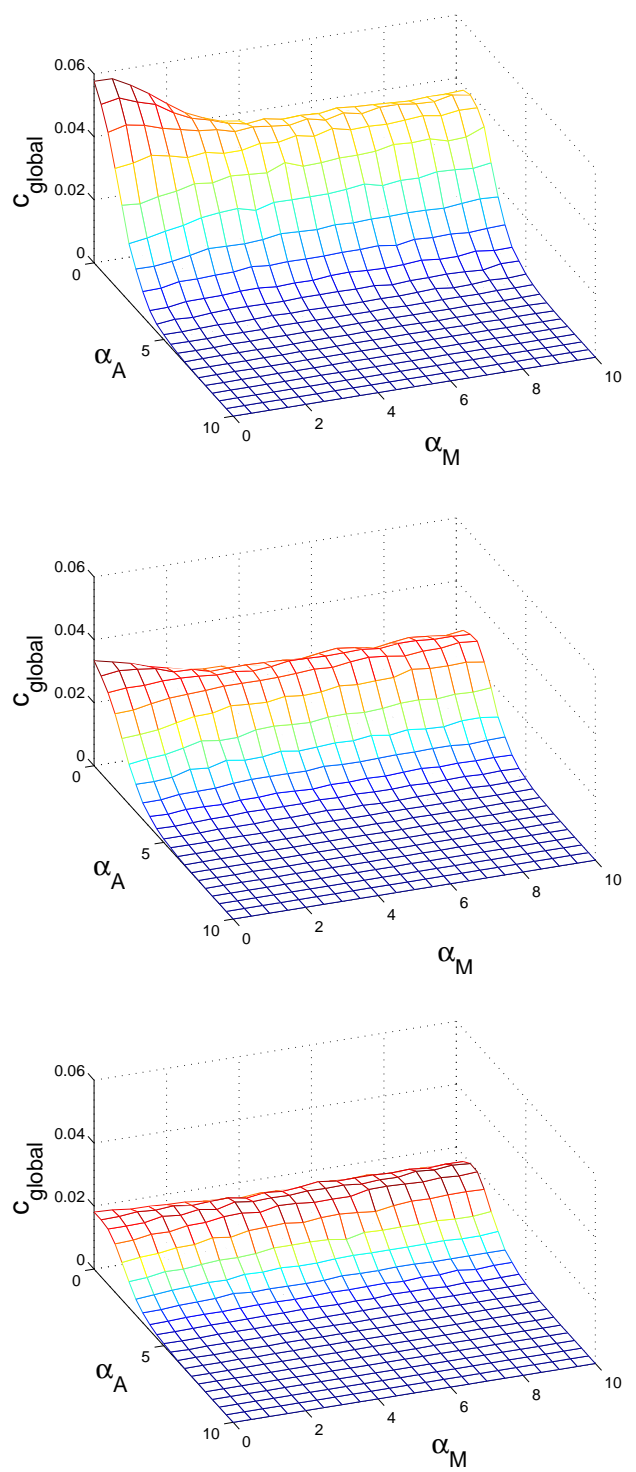

\section{Random}
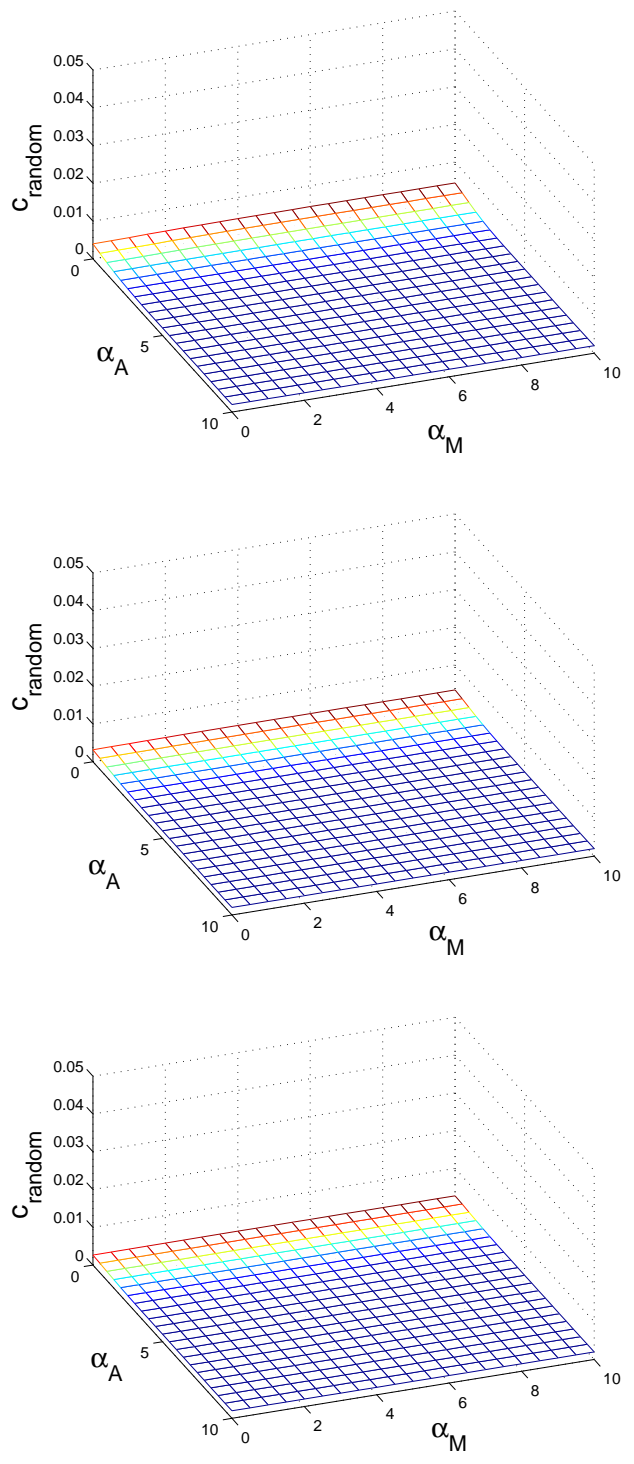

FIG. 8: Global clustering coefficient in the $\alpha_{A}-\alpha_{M}$ plane for $\lambda=0.5,1,2$ (from top to bottom) and $N=1000$ for the present model (a) and for an ER random graph with the same number of links and nodes (b). Averages were taken over 100 independent realizations.

[7] D.R. White, N. Kejzar, C. Tsallis, J.D. Farmer, and S. White, Phys. Rev. E 73, 016119 (2006).

[8] M.D.S. de Meneses, S.D. da Cunha, D.J.B. Soares and L.R. da Silva, in Complexity and Nonextensivity: New Trends in Statistical Mechanics, eds. M. Sakagami, N. Suzuki and S. Abe, Prog. Theor. Phys. Suppl. 162, 131-137 (2006).

[9] H. Hasegawa, Physica A 365 383-401 (2006).

[10] S. Boccaletti, V. Latora, Y. Moreno, M. Chavez and D.-U. Hwang, Phys. Rep. 424, 175-308 (2006).

[11] C. Tsallis, R.S. Mendes, and A.R. Plastino, Physica A 261, 534-554 (1998).

[12] A.-L. Barabasi and R. Albert. Science, 286, 509- 512 (1999).

[13] R. Albert and A.-L. Barabasi, Phys. Rev. Lett. 85, 5234-5237 (2000).

[14] B.J. Kim, A. Trusina, P. Minnhagen, and K. Sneppen, Eur. Phy. J. B 43, 369 (2005).

[15] S. Abe and S. Thurner, Phys. Rev. E 72, 036102 (2005).

[16] C. Beck and E.G.D. Cohen, Physica A 322, 267-275 (2003).

[17] M. Baiesi and S.S. Manna. Phys. Rev. E, 68, 047103 (2003).

[18] G. Palla, I. Dernyi, I. Farkas, and T. Vicsek, Phys. Rev. E 69, 046117 (2004); I. Farkas, I. Dernyi, G. Palla, and T. Vicsek, Lect. Notes in Phys. 650, 163-187 (2004). 
[19] J. Park and M.E.J. Newman, Phys. Rev. E 70, 066117 (2004).

[20] C. Biely and S. Thurner, cond-mat/0507670.

[21] E. Ravasz and A.-L. Barabasi, Phys. Rev. E 67, 026112 (2003).

[22] P. Erdös and A. Rényi, Publ. Math. Debrecen 6, 290-297 (1959); Publ. Math. Inst. Hung. Acad. Sci. 5, 17-61 (1960).

[23] D.J. Watts and S.H. Strogatz, Nature 393, 440 (1998). 